\title{
La place de la mémoire dans les aménagements territoriaux, un enjeu géopolitique.
}

The place of memory in the territorial arrangements. A geopolitical issue Der Ort der Erinnerung in territorialen Regelungen. Eine geopolitische Frage.

Pierre Ginet and Laurène Wiesztort

\section{(2) OpenEdition}

\section{Journals}

Electronic version

URL: http://journals.openedition.org/rge/5059

DOI: $10.4000 /$ rge.5059

ISSN: 2108-6478

\section{Publisher}

Association des géographes de l'Est

\section{Printed version}

Date of publication: 30 December 2013

ISSN: 0035-3213

\section{Electronic reference}

Pierre Ginet and Laurène Wiesztort, « La place de la mémoire dans les aménagements territoriaux, un enjeu géopolitique. », Revue Géographique de l'Est [Online], vol. 53 / 3-4 | 2013, Online since 02 July 2014, connection on 08 September 2020. URL : http://journals.openedition.org/rge/5059 ; DOI : https://doi.org/10.4000/rge.5059

This text was automatically generated on 8 September 2020

Tous droits réservés 


\section{La place de la mémoire dans les aménagements territoriaux, un enjeu géopolitique.}

The place of memory in the territorial arrangements. A geopolitical issue

Der Ort der Erinnerung in territorialen Regelungen. Eine geopolitische Frage.

Pierre Ginet and Laurène Wiesztort

\section{Introduction}

1 Le lien entre la mémoire et sa prise en compte dans la production ou la recomposition territoriale ne relève pas de l'évidence, encore moins d'un simple et innocent souci esthétique ou conservatoire. Le choix de mise en valeur de tel ou tel équipement, site ou portion d'espace plus ou moins vaste traduit a priori l'adhésion d'une majeure partie du groupe social qui initie cette action, aux valeurs historiques et culturelles incarnées par ces objets géographiques patrimoniaux : tel village, que l'on s'attend à voir se rassembler devant le monument aux morts chaque 11 novembre, ou la population de la planète qui reconnaît le bien fondé des classements opérés par l'UNESCO au titre du patrimoine mondial de l'Humanité...

2 Force est de constater que l'évidence de politiques mémorielles fédératrices est bousculée par un ensemble de changements majeurs intervenus au cours des dernières années. La mondialisation brasse désormais des populations extrêmement diverses à tous les échelons géographiques, de la ville, par le biais de la métropolisation, à la planète toute entière, affectée par des processus de globalisation. Cela peut remettre en question les codes sociaux ancrés dans des formes de territorialités anciennes, et l'adhésion aux valeurs mémorielles prises en compte dans les processus de production et de recomposition qui les concernent.

De nouveaux rapports de force bouleversent ces fragiles équilibres. Adolescents et personnes âgées, immigrés et autochtones, travailleurs et inactifs, riches et pauvres, les 
populations ne se reconnaissent plus toutes nécessairement dans les objets mémoriels valorisés, voire en considèrent certains comme opposés à leurs propres valeurs voire même leurs identités. Le concept d'identité développé en psychologie sociale correspond à l'image que nous avons de nous-mêmes en fonction des contextes sociaux dans lesquels nous vivons et des apprentissages sociaux dans lesquels nous sommes impliqués. L'identité est le tissu qui nourrit nos désirs et nos valeurs et les construits en une image de nous-mêmes désignée sous le terme d'identité personnelle ou de Soi. Il s'agit d'un processus psychologique de représentation qui se traduit par le sentiment d'exister en tant qu'être singulier et également d'être reconnu comme tel par autrui (Fischer G-N, 2005). La question de l'identité est essentielle, elle doit être prise en compte dans les processus d'élaboration des projets urbains. Plusieurs questions peuvent être posées: quelles identités locales et sociales sont sollicitées dans la conception du projet urbain et sur quelles constructions reposent-elles? En quoi la mobilisation identitaire participe-t-elle au processus de conception du projet, puis à son appropriation? Mais aussi quel rôle va jouer le projet urbain dans l'élaboration identitaire? (Hayot A., Sauvage A., 2000).

De nos jours, la discordance des mémoires et donc des identités s'exacerbe. La prise en compte de telle référence mémorielle peut alors catalyser les clivages socioterritoriaux et dégénérer en tensions ou clivages en France, la fonction symbolique de la mémoire - symbolo: qui rassemble - jouant dans ce cas un rôle diabolique! (étymologiquement diabolo: qui sépare). Le rythme accéléré et permanent des recompositions sociopolitiques interagit désormais avec le temps long des objets mémoriels (héritages détachés des différents contextes socio-économiques et politiques). Ces recompositions englobent l'ensemble des changements continus qui vont de la définition des frontières du pays, à la réforme en cours de l'intercommunalité mais aussi l'ensemble des modes de gouvernance, d'administration et de gestion. Le choix de la ou des mémoires qui sont et seront prises en compte dans l'aménagement apparaît de façon de plus en plus évidente comme un enjeu politique et géopolitique majeur. La mémoire correspond au souvenir, ou l'ensemble des souvenirs, conscients ou non, d'une expérience vécue et/ou mythifiée (Nora P., 2011), à ne pas confondre (comme nous le verrons) avec l'Histoire ou le patrimoine.

La mémoire constitue bien un objet d'étude pour le géographe et ne se résume pas à la seule question de l'inventaire et de la préservation du patrimoine. Le patrimoine étant un bien commun reçu des siècles et des générations passés. Il est également un enjeu, dont la maîtrise dépend de chacun. Cette notion fait appel à l'idée d'un héritage légué par les générations antérieures et qu'il faut transmettre aux générations suivantes et dépasse les limites de la propriété (Benhamou, 2012).

\section{Les dispositifs juridiques de prise en compte de la mémoire dans l'aménagement}

Que l'instrumentalisation de la mémoire passe par sa valorisation, son oubli ou son effacement, cette première constitue dans tous les cas un acte politique. Son traitement s'inscrit dans une géographie politique en perpétuelle reconstruction. Sa mise en œuvre, implicite ou explicitée dans des programmes plus ou moins ambitieux, relève donc de la géopolitique et d'une géostratégie plus ou moins ostentatoire ou plus ou moins masquée. L'analyse des présupposés idéologiques qui en fondent 
l'instrumentalisation devient dès lors nécessaire. Une telle approche permet d'enrichir l'analyse fondatrice mais ancienne, proposée dès les années 1960 (Choay, 1965). Deux principales postures, intégrant implicitement une partie de la question de la mémoire, y étaient décrites. Une première, regroupant les urbanistes dans le courant des progressistes, innovateurs rejetant la mémoire et tournés vers un futur à inventer, et une seconde dans celui des culturalistes, tenant d'un passé source d'inspiration et de modèles à retrouver par un effort de mémoire.

\section{A. La mémoire, champ de bataille idéologique?}

7 La mémoire est longtemps apparue comme une préoccupation purement philosophique ou psychologique. La mémoire individuelle est souvent pensée sans référence aucune à la société, voire au groupe auquel l'individu appartient; Elle peut être considérée comme un attribut de la personne, voire un de ses fondements, ce qui définit au mieux sa personnalité. Jean-Yves et Marc Tadié (1999) expose dans leur ouvrage, l'idée que la fonction de la mémoire est de nous permettre de nous reconnaître en tant qu'être unique (avec son vécu et son quotidien). Ainsi, c'est la mémoire qui donne sa cohérence et son unicité à notre personnalité.

8 Jusque dans les années 60 , le discours public sur le passé était dominé par le quasi monopole de l'histoire. Puis sont apparus de nouveaux discours qui affirmaient avoir une valeur historique : ceux de la mémoire, et plus particulièrement de la mémoire collective de groupe. On parlera notamment de la mémoire collective des survivants d'expériences de destruction tels que les Juifs de la diaspora. Une mémoire qu'il est parfois difficile à faire émerger, devant parfois fissurer "un mur de silence " (Benjamin Stora, 1999). Pour qu'une mémoire collective se constitue publiquement, il faut un travail du groupe sur lui-même. Il est souvent difficile de s'approprier un passé douloureux, de transformer une mémoire collective aussi, individuelle et/ou familiale en histoire. Aujourd'hui, malgré une production historiographique considérable, Michel Wieviorka (2001) explique que la mobilisation mémorielle s'effectue en ordre dispersé, sur des enjeux diffus et contradictoires. Le travail de la mémoire est très complexe, car elle est très souvent rattachée à des sentiments de perte, de destruction. Elle va ainsi manquer d'objectivité. La mémoire n'est pas l'histoire. L'histoire est une discipline du savoir qui se réclame de la raison, de la science, alors que la mémoire relève plutôt des affects, de la conscience, des émotions, des sentiments, des passions. Pierre Nora (2004) le souligne lorsqu'il affirme « la mémoire est ouverte à la dialectique du souvenir et de l'amnésie, inconsciente de ses déformations successives, vulnérable à toutes les utilisations et manipulations, susceptibles de longues latences et de soudaines revitalisations ». La mémoire est une sélection, puisque certains traits de l'évènement seront conservés, d'autres écartés (progressivement ou rapidement, volontairement ou non)puis oubliés (Todorov T., 1995). L'histoire a également la caractéristique de s'appuyer sur la raison, contrairement à la mémoire, dans le sens où elle appelle à la démonstration, à la preuve, à la comparaison des écrits, des récits, des objets matériels et des images. Ainsi l'histoire, à première vue, est plus objective que la mémoire pour laquelle il est possible de remettre en cause sa crédibilité. Pour autant depuis les années 60 , de nombreux groupes de mémoires collectives remettent quand à eux en cause l'histoire. L'histoire dans son universalisme nécessaire, peut parfois oublier de s'interroger sur la subjectivité de ceux qui la font... pouvant même parfois être sélective. Tzvetan Todorov (1995) expose le fait qu'il peut parfois y avoir une 
nécessaire occultation d'actions jugées pourtant essentielles qui va conduire à des positions paradoxales, il résume son idée à travers un propos tenu par Himmler lors du Procès des grands criminels de guerre devant le tribunal militaire international à Nuremberg en 1947 à propos de la "solution finale" : «C'est une page glorieuse de notre histoire, qui n'a jamais été écrite et ne le sera jamais». Il est pourtant question d'un devoir selon Todorov, lorsque les événements vécus par un ou des individus sont de nature exceptionnelle ou tragique, ce droit devient un véritable devoir, celui de témoigner.

9 L'évidence de la valorisation du patrimoine peut masquer des stratégies d'affirmation identitaires portées par des leaders d'opinion et conditionnant les conditions de reproduction ou de production de schémas d'organisation socio-territoriaux. Le terrain de la mémoire, présent en premier lieu dans la notion de patrimoine, apparaît comme un champ de bataille idéologique entre différents modèles et projets de société.

\section{B. Une organisation multiscalaire qui traite de patrimoine... et évite de parler de mémoire, de valeurs et de politique}

Posons immédiatement les différences entre les deux notions : patrimoine et mémoire. Le concept de patrimoine a beaucoup évolué et il ne s'applique qu'à un certain nombre de biens particuliers à qui l'on reconnait une valeur spécifique d'un point de vue historique, artistique, esthétique, de la mémoire collective ou du témoignage (IAURIF, 2000). Cette reconnaissance est surtout propre aux membres des catégories dominantes de la société (Amougou E., 2004) occupant des positions légitimes, notamment dans les instances décisionnelles de l'Etat, caractérisées par le monopole des définitions de ce qui doit être ou non considéré comme du patrimoine. Ressort ici l'idée de la sélection. C'est ce qui peut différencier le patrimoine de la mémoire, les individus peuvent faire part de leurs mémoires, de leurs souvenirs, sans que nous puissions (hormis les pressions dont traite très bien Todorov dans son ouvrage en 2000) empêcher véritablement aujourd'hui la diffusion (via les nouveaux moyens d'expressions: réseaux sociaux, internet).

11 Le patrimoine a longtemps été réduit aux seuls monuments historiques, mais il a peu à peu perdu son caractère privé devenant dans les esprits un héritage collectif, dont chacun doit se porter garant. L'institut d'aménagement et d'urbanisme de la région Ilede-France (2000) souligne qu'il n'y a pas un Patrimoine mais des patrimoines, que tout semble devenir du patrimoine au point que nous finissons par confondre cette notion avec l'idée de témoignages ou de mémoire.

12 Enfin, dans sa définition Dominique Audrerie (2003) cite l'abbé Grégoire, dans l'un de ses rapports à la Convention qui explique que le patrimoine et plus particulièrement les objets nationaux n'étant à personne, sont la propriété de tous (ce qui n'est pas le cas de la mémoire qui est propre à chaque individu). L'Unesco insiste sur la double dimension du patrimoine, culturelle et naturelle, toutes deux en étroite relation. La première reste la base de notre législation. Ce patrimoine correspond à l'œuvre de l'homme au fil des siècles, il témoigne alors du génie d'une nation. Enfin le patrimoine peut être matériel ou immatériel. Il ne se limite pas aux bien matériels, meubles, immeubles, sites, mais il intéresse aussi les langues, les coutumes, les savoir-faire. La mémoire ne fait pas partie de ce patrimoine immatériel, c'est là que se pose la grande différence entre les deux notions : patrimoine et mémoire, souvent confondues. 
13 Il ne s'agit pas ici de présenter la liste chronologique des textes de loi en matière de protection du patrimoine mais de constater la mise en place d'un cadre juridique très complet autour de la question de la mémoire, abordée au travers de cette question du patrimoine matériel et immatériel. Ce cadre juridique conceptualise et borne l'objet mémoire dans des limites que peu de géographes ont franchies jusqu'à présent.

Ce cadre s'est organisé au cours des dernières décennies. Il définit une acception restreinte de la notion de mémoire à celle de patrimoine, au travers de la question de son identification, de son inventaire, de sa sauvegarde et de sa valorisation. Ce cadre est présent à différentes échelles géographiques, emboîtées, de l'échelon mondial (UNESCO, 1972) à l'échelon européen (Journées européennes du patrimoine depuis 1991, Convention européenne sur le patrimoine archéologique de 1992) puis à l'échelon national (Ainsi en France l'ensemble des dispositifs juridiques nationaux depuis l'invention de la notion de patrimoine et la mise à la disposition des biens de l'Eglise de la Nation par décret de l'Assemblée constituante en 1789) et enfin à l'échelon local (les collectivités territoriales sont souvent initiatrices de démarches de patrimonialisation).

La formation de ce cadre juridique est partie du niveau national pour se diffuser à l'échelon international (la France a joué un rôle moteur: le siège de l'UNESCO ne se trouve pas par hasard à Paris) puis au niveau local. Notre hypothèse est que ce cadre juridique, qui garantit a priori une prise en compte de la "diversité culturelle" (UNESCO, 2002), par ses critères de sélection des sites, les modalités de participation du public à ces questions..., n'empêche pas l'existence d'un ensemble de postures idéologiques dans lesquelles la notion de patrimoine est un masque destiné à protéger ou à conforter l'essor de ces dernières.

\section{La mémoire comme terrain de recherche pour les géographes}

\section{A. Les géographes sont très peu présents sur la thématique de la mémoire}

Le patrimoine fait l'objet de nombreux travaux de recherche (Beghain P., Bortolotto C., Choay F., Heinich N., Patin V., etc.), avec un angle classique qui est celui de la sauvegarde, abordée essentiellement sous son aspect procédural, comme un élément parmi d'autres du projet urbain. Par contre les géographes se sont très peu investis dans des recherches portant sur l'instrumentalisation politique de la mémoire dans le processus permanent de recomposition urbaine et territoriale. Certains se sont risqués à travailler sur la mémoire urbaine, au travers d'une analyse d'enjeux sociaux liés à la patrimonialisation et à la démolition (Veschambre, 2008), mais en passant sous silence les implications politiques soulevées par ces questions.

17 Sur le site Géomonde (PRODIG, 2012), sur un total de 1.634 notices individuelles, 14 géographes font état de la thématique "mémoire » tandis que 88 font apparaitre la thématique "patrimoine »... Cette répartition traduit un faible investissement sur la thématique mémorielle en général et, lorsqu'on examine plus en détail les champs de recherche recouverts par la thématique "patrimoine", on remarque une entrée préférentielle sur l'approche classique de la préservation du bâti. Par exemple, dans plusieurs articles d'Hérodote ou de la Revue Géographique de l'Est tel que celui de 
Simon Edelblutte (2009) qui traitent de la conservation artificielle d'éléments patrimoniaux qui permettent de préserver la mémoire d'un site industriel, d'un quartier, ou de projet de recompositions urbaines réalisées sur des aspects identitaires et mémoriels. Ou l'approche de territoires sensibles sous tensions (Irlande du Nord, ExYougoslavie). Les travaux de Maria Gravari-Barbas partent de l'idée d'appropriation des territoires mais aussi d'inégalités d'appropriation, qu'elle observe à travers le patrimoine et en posant les questions suivantes: Qui dit patrimoine dit objet, monument, espace, mais aussi référence partagée, identité et mémoire collectives, bien commun. Mais peut-on réellement considérer qu'il y a toujours et partout appropriation collective ? Pourquoi le patrimoine suscite t-il tant de conflits? (GravariBarbas, 2010).

Les autres entrées sur la question ont finalement été abandonnées à quelques historiens, dont le créateur des "lieux de mémoire " (Nora, 1997), aux architectesurbanistes, parmi lesquels l'initiateur d'une psychologie des représentations, appliquée à la production urbaine (Lynch, 1969)), ou à des spécialistes d'information et de communication traitant de "mémoire sociale» (Moles, 1969) en relation avec la question "des densités de répartition des équipements culturels "(Moles, 1969). Les géographes l'ont plus évoquée qu'ils ne s'en sont collectivement emparés. Ainsi lorsque S.Rosière cite V.Draskovic, ancien Président Serbe du renouveau affirmant au début des années 1990 «il faut se battre pour toutes les terres de Yougoslavie où se trouve un cimetière Serbe " (Rosière, 2007), il montre les enjeux lié à la thématique. Mais le terrain demeure celui de grands écrivains (Proust), parmi lesquels figure au moins un géographe (Gracq, 1989)! Nous pourrions également citer Yves Lacoste qui fait de l'étude des représentations la base de sa démarche géopolitique. L'idée de représentation chez Lacoste se construit en grande partie sur la mémoire, et justement sur l'instrumentalisation et la réécriture de l'Histoire. Comme Pierre Nora, il expose l'idée d'une Histoire déformée et l'existence de flous et d'imperfections qui caractérisent souvent la mémoire. 


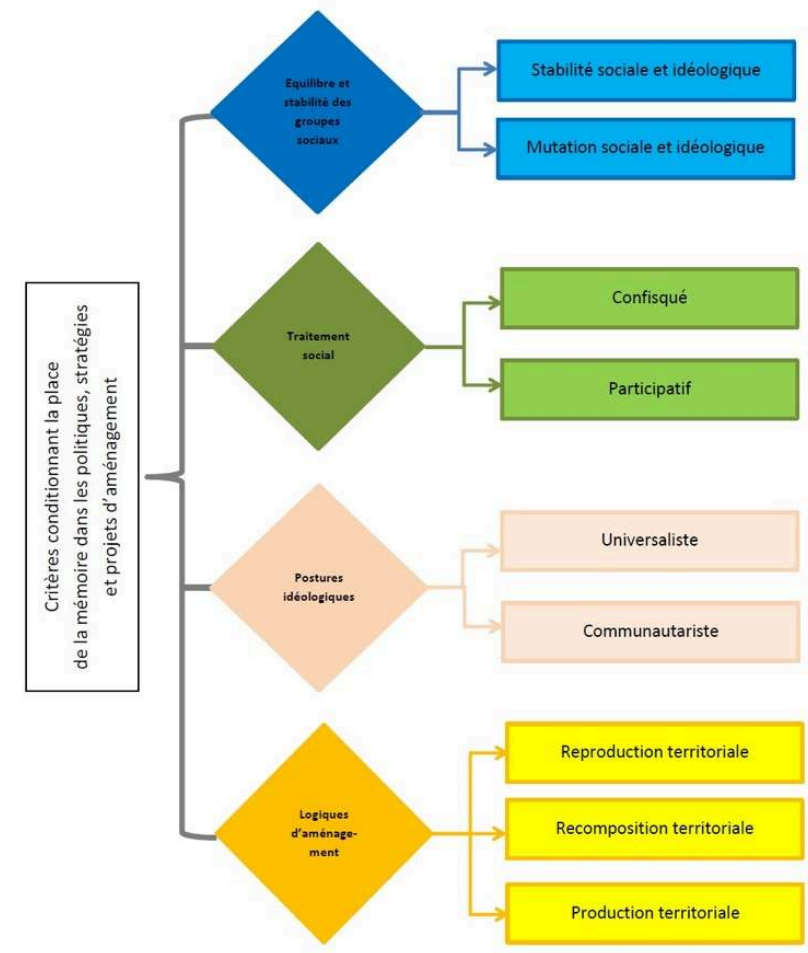

Document original réalisé par Ginet $P$.

\section{B. Les enjeux sociétaux et géopolitiques sont pourtant considérables}

Jusque dans les années 1960, la croyance au progrès aborde le passé de façon négative et le rejette. La "civilisation» peut et doit nécessairement le surmonter pour s'accomplir, a fortiori dans le cadre urbain qui s'impose à une part croissante d'entre elle. Ainsi avec Le Corbusier et les fonctionnalistes, les acteurs de l'aménagement font table rase du passé pour projeter l'homme dans le seul avenir qui semble souhaitable au travers de nouveaux matériaux, de nouvelles techniques de construction, de nouveaux concepts et modèles architecturaux. Les approches modélisatrices et normatives ont le vent en poupe. Les opérateurs territoriaux détruisent des centres villes (Liège StLambert dans les années 1970, Lille St-Sauveur dans les années 1950, etc.) ou manquent de peu de passer à l'acte (Quartier des Marolles à Bruxelles en 1969)... Il s'agit de défaire l'homme des scories, pesanteurs, artifices et contingences du passé pour moderniser son espace de vie. Les années 1950 puis 1960 font la part belle à la Résorption de l'Habitat Insalubre (RHI), à la Rénovation Urbaine (RU) dans sa première version, fondée sur le curetage urbain, l'élimination des dents creuses, l'expropriation et la reconstruction à neuf pour de nouvelles fonctions ou de nouvelles populations.

Aujourd'hui, les références au passé, à l'histoire, à la mémoire, se multiplient. La prise en compte de la mémoire et de l'histoire a connu une accélération continue depuis les années 1960. De la Loi Malraux sur les secteurs sauvegardés de 1962 aux Plans de Sauvegarde et de Mise en Valeur (PSMV) des Plans Locaux d'Urbanisme (PLU) actuels, de la rénovation de cités minières à la création de l'Agence Nationale de la Rénovation 
Urbaine (ANRU) sans oublier la multiplication des projets de classement de l'Organisation des Nations Unies pour la Science, l'Education et la Culture (UNESCO) Langres par exemple, acropole située sur un éperon et ceinte par $8 \mathrm{~km}$ de fortifications, labellisée «Ville d'art et d'histoire » depuis 1985, se propose de relever en 2011 le défi de concourir au patrimoine mondial de l'UNESCO et ainsi faire reconnaître sa valeur universelle - ou la prise en compte du patrimoine dans le Code de l'Urbanisme : mise en place des Zones de Protection du Patrimoine Architectural, Urbain et Paysager (ZPPAUP) en 1983 remplacées par les Aires de mise en Valeur de l'Architecture et du Patrimoine (AVAP) depuis la Loi Grenelle II de 2010... Cette juridisation d'une mémoire réduite au patrimoine s'accélère.

21 Les enjeux soulevés par ce regain d'intérêt voire ce renouveau «culturaliste » (Choay, 1965) sont d'abord d'ordre politique. Ils relèvent selon Béatrice Giblin de la "géopolitique interne» (Lacoste, 1995) ou mieux de la "géopolitique urbaine » (Hulbert, 2009). Aujourd'hui, nous sommes face à des «collections » de territoires locaux et de populations locales aux sorts tragiquement différents qui engendrent des complexités au sein d'identités collectives plus nombreuses (Subra P., 2007). Mettre en valeur telle ou telle mémoire ou plus exactement, la manipuler, l'instrumentaliser voire la réviser (dans l'acception la plus maligne du terme), devient une façon d'intervenir sur les territoires en cours de production, de reproduction ou de recomposition. Françoise Benhamou (2012) parle de « biens générateurs d'externalités » biens souvent positives (par exemple : la proximité avec un monument classé crée de la valeur pour les autres habitations qui l'entourent, de la fierté, une amélioration du cadre de vie, etc.). Loin du folklore ou de l'anecdote, cette prise en compte de la mémoire revêt une importance essentielle dans la manière dont tout projet territorial se formule, se construit puis se déroule au cours du temps. La mémoire relève finalement moins de l'espace vécu que de l'espace voulu et traduit moins une vision rétrospective qu'une vision prospective.

La mise en valeur de la mémoire fait partie intégrante de tout projet de territoire. De même, la mémoire ou création d'une mémoire peut revaloriser un quartier. Dans le cas où le passé est peu connu ou qu'il n'y pas de réelle histoire associée à un lieu, alors un hommage peut être rendu à une personnalité, à un événement historique par le biais d'un nom de rue, de quartier, d'une statue, d'une œuvre d'art, sans lien évident avec le site d'accueil. C'est ici une stratégie toponymique de valorisation territoriale. Le premier réflexe des découvreurs de terres (Magellan, Vasco de Gamma...) était de baptiser leurs découvertes et d'inscrire leur nouveau nom sur une carte. Ici il s'agit d'introduire l'histoire et les valeurs sociales dans la réalité géographique. On s'aperçoit que lors des situations de conflits, changer les noms devient prioritaire (cf. les noms de communes en breton plutôt qu'en français, en néerlandais plutôt qu'en wallon dans les communes belges autour de Bruxelles ou près de la frontière linguistique séparant les deux Communautés).

On s'en aperçoit en constatant que la mémoire, dans la production/recomposition territoriale et son corollaire l'aménagement du territoire, est sélective. Aussi le choix politique d'opter pour un pan ou un élément de l'histoire plutôt qu'un autre n'est ni neutre ni innocent. Ceci est d'autant plus visible que ce choix peut être explicité et démocratiquement partagé ou au contraire masqué et confisqué par une élite. Dans tous les cas, l'instrumentalisation ou la mise en scène de la mémoire dans l'acte d'aménager l'espace contribue à conduire le territoire dans une direction qui exprime 
la déclinaison géographique d'un choix politique. Ces muséification ou la conservation d'une mémoire dans un projet urbain, un aménagement spécifique du territoire répondent également à une demande sociale, «Des français de plus en plus nombreux semblent aujourd'hui inquiets de perdre, avec leur mémoire, leur identité, leur identité collective, c'est-à-dire, en fait, le faisceau de leurs identités de groupes " (Crubellier, 1991). C'est donc aussi une stratégie politique. A l'inverse, un aménagement ou une muséification peut également influencer, modifier l'image d'une ville ou d'une région de manière réductrice. Prenons l'exemple de la ville de Vimy (Nord-Pas-de-Calais) qui est reconnue aujourd'hui en France et à l'étranger uniquement pour son Parc mémorial canadien qui est le plus prestigieux des monuments canadiens d'Europe qui rend hommage à ses citoyens qui ont combattu et donné leur vie au cours de la première guerre mondiale. La ville a perdu sa propre identité.

Pourtant les enjeux liés au brassage des populations et des valeurs (Obin, 2004) s'exacerbent, dans le contexte de la mondialisation. La concurrence entre sociétés humaines pour imposer leurs valeurs à l'échelle de la planète est patente. L'émergence de modèles de société justifiés par une mémoire, vient en concurrencer d'autres. Même la Déclaration Universelle des Droits de l'Homme et du Citoyen, adoptée par les Nations Unies en 1948 est parfois rejetée car considérée comme occident-centrée... Aux références à l'histoire de la Révolution s'opposent des références à d'autres histoires, jugées porteuses de valeurs plus universelles. L'historien Nicolas Trifon a appelé " engrenage de la concurrence mémorielle » le danger qu'encourt une société à ce que des groupes d'individus revendiquent des attentions particulières en invoquant des drames humains ayant touché par le passé leurs communautés d'origine (Trifon, 2008). Régis Debray, philosophe français, réagira à une proposition de Nicolas Sarkozy de " confier la mémoire d'un enfant juif déporté à chaque écolier de dernière année d'école primaire ». Il estime alors que, certes, le devoir de mémoire est nécessaire mais il ne faut pas que cela devienne un abus. Il expliquera que selon lui, il y a un risque d'escalade, de surenchère, de concurrence des mémoires. Pourquoi et comment choisir entre la mémoire d'un enfant juif et celle d'une jeune victime d'Israël en Palestine ? Il y a un risque de polémique voire de division selon les clivages communautaires et confessionnels. Le combat pour la reconnaissance de valeurs implique une reconnaissance de mémoires passées sous silence, et leur affirmation dans la géographie des lieux et des espaces géographiques à toutes les échelles. La concurrence des valeurs est une concurrence des mémoires. L'enjeu est politique, son fondement est moral. Les enjeux en termes de cohésion socio-territoriale sont considérables. Yves Lacoste (1995) parle de « la nation et des représentations concurrentes » et il fonde son propos sur un exemple, le fait que récemment dans les pays d'Europe occidentale mais aussi dans le monde musulman, l'Etat-nation n'est plus la seule représentation géopolitique et se trouve alors en concurrence avec d'autres représentations, elles aussi chargées de valeurs. Leur diffusion est le fait d'intellectuels (ex: les mouvements islamiques qui souhaitent appliquer la loi coranique). De plus, en Europe occidentale, dans un certain nombre de vieux Etats-nations, les valeurs associées à la nation sont parfois considérées comme dépassées et l'identité régionale cherche à s'imposer. 


\section{Détermination de la valeur des mémoires et choix politiques}

\section{A. La détermination de la valeur et les modes d'action}

La mémoire est sélective, également en aménagement. Sa prise en compte dans les politiques, stratégies et projets d'aménagement dépend de la valeur accordée aux objets, en l'occurrence géographiques, par les décideurs, en fonction des rapports entre groupes sociaux occupant l'espace. C'est un point largement évoqués dans les études et nombreux ouvrages sur le patrimoine, celui-ci devenant même parfois un patrimoinealibi. Pour Maurice Crubellier (1991) «Mémoriser c'est, pour un groupe, se constituer un patrimoine de souvenirs, valoriser, voire survaloriser des personnages, des événements, à l'exclusion d'autres, c'est réaliser une appropriation sélective du passé ». Les objets considérés comme ayant de la valeur occuperont une place centrale dans les politiques d'aménagement (ex.: Fosse Delloye à Masny devenue Centre Historique Minier régional). Certains feront même l'objet d'une véritable sanctuarisation (Ossuaire de Douaumont, Mur des lamentations à Jérusalem). Les objets jugés sans valeur seront oubliés, abandonnés à leur sort puis réinvestis une fois que leur délabrement puis leur identification en tant que friche les aura définitivement ouverts à un recyclage fonctionnel ; Jean Chesneaux explique ainsi que «La mémoire urbaine se dégrade volontiers en ville-musée " (Chesneaux, 2001). Les objets contraires aux valeurs seront quant à eux détruits. La première et la troisième posture (valoriser, détruire) sont actives. Parler de mémoire dans l'aménagement c'est par conséquent d'abord parler de valeur. Selon Marie-Hélène Bacque et Sylvie Fol dans leurs contributions à un colloque consacré au projet Urbain en février 1997 (Hayot, 2000), la question des valeurs identitaires apparait de manière récurrente et constitue aujourd'hui un enjeu central dans les projets urbains. Se dessine une géographie vécue, voulue, perçue de la valeur ou du moins de la représentation à un moment donné par un groupe social plus ou moins étendu, de ses valeurs. Cette détermination de la valeur mémorielle exprime par conséquent aussi un ensemble de rapports de force entre groupes sociaux. Elle peut privilégier une ou plusieurs dimensions de la valeur : valeur marchande ou non marchande, estimable ou inestimable, sacrée ou profane, de portée locale ou de portée universelle, d'usage ou d'échange (fixée par le marché), symbolique ou matérielle, etc. 


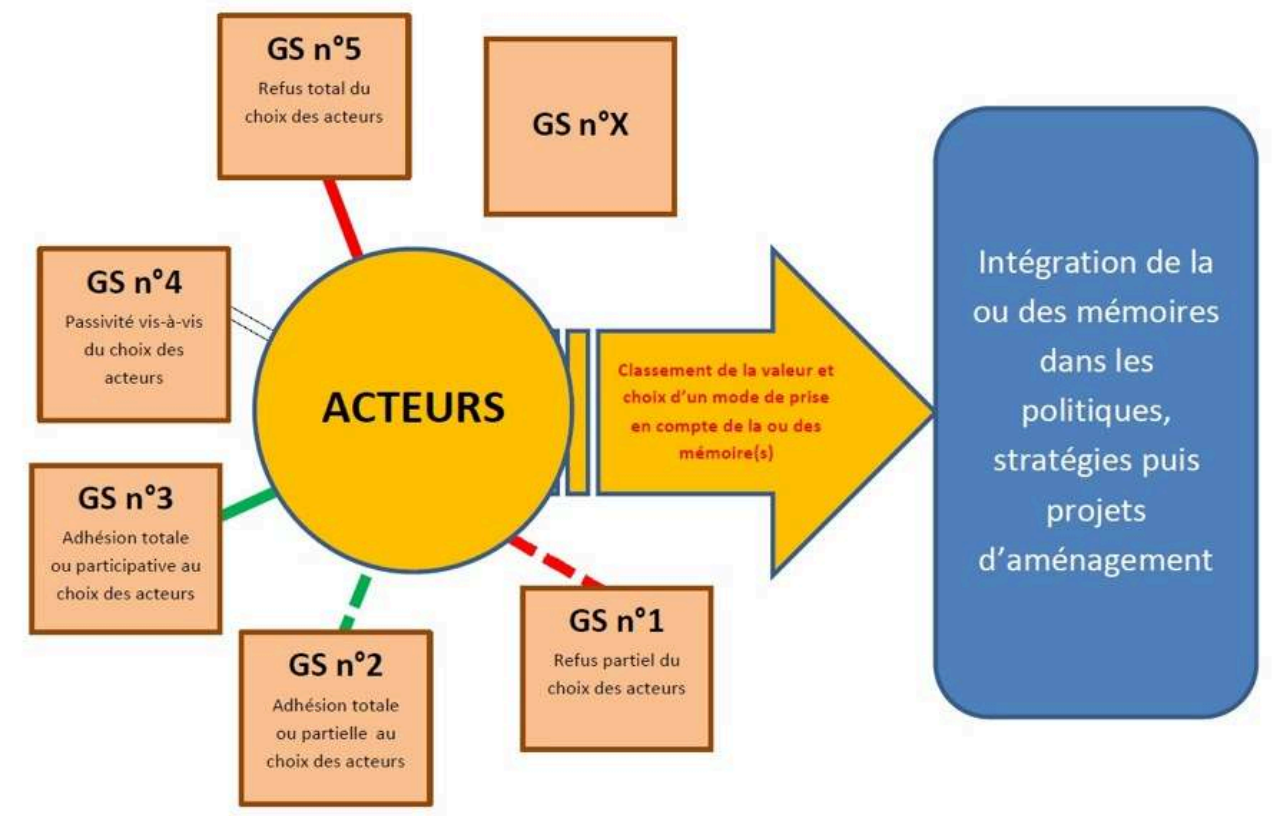

Document original réalisé par Ginet P.

\section{B. Les acteurs qui portent la sensibilité mémorielle}

Les acteurs sont nombreux, les associations, services ou agents de l'Etat, collectivités, professionnels (artisans, architectes, etc.), fondation, particuliers etc. Chacun d'entre eux est un vecteur permettant l'expression de la sensibilité de la société à la mémoire, au patrimoine. Ils expriment alors l'attachement que la société ressent et montre pour une période de l'Histoire, un événement, un souvenir, un témoignage, un personnage. Les associations par exemple, tiennent une place particulière, puisqu'elles ont le pouvoir d'influencer les décisionnaires de la conservation ou la destruction (choix d'oublier) de telle ou telle mémoire, souvent par le biais de pressions ou tout simplement de manifestations d'information et/ou de sensibilisation (spectacles vivants, films, séminaires) telles que les Amis de la Fondation pour la Mémoire de la Déportation (AFMD) qui regroupent toutes les personnes physiques ou morales qui souhaitent agir pour assurer la pérennité, l'enrichissement et la transmission de la mémoire française et européenne de l'Internement et de la Déportation. Les pressions pour conserver une mémoire peuvent être parfois plus radicales sous la forme de pétitions ou de lutte acharnées entre les membres des associations et les forces de l'ordre. Nous nous sentons tous porteur d'une mémoire que nous cherchons à transmettre car elle conforte notre sentiment d'identité et donnent sens à la trajectoire de nos vies collectives autant qu'individuelles, par-delà la succession des générations (vécu des anciens, souvenirs familiaux, coutumes, d'ordre privé ou ayant été affectés directement ou indirectement par un événement marquant de l'Histoire). 


\section{Les différentes postures politiques de prise en compte de la mémoire dans les projets d'aménagements}

voire le caractère unique d'un territoire. L'ouverture des frontières, l'internationalisation des sociétés et des économies contribuent à une mobilité croissante des personnes, des capitaux et des représentations. Le Président de Communauté Urbaine ou le Maire «bâtisseur » vont alors pouvoir utiliser la mémoire comme un moyen de spécifier leur territoire, de le rendre plus visible sur la carte d'une région, d'un état ou du monde. Ces démarches constituent aussi des réponses à l'épuisement de modèles de développement et d'aménagement ancrés dans une postmodernité qui s'essouffle. Ces modèles présentés comme universels, encore triomphants il y a quelques années et presque copiés-collés à l'infini, sont désormais à l'origine de faillites retentissantes et de plus en plus nombreuses. C'est le cas du krach de villes espagnoles, construites pour rien avec l'appui financier et moral des systèmes bancaire et politique (El Quiñon), ou l'exemple de la ville nouvelle de Kilamba, en Angola, devenue à peine construite une ville fantôme... sont autant de contre-exemples qui incitent à s'interroger sur l'intérêt de recourir davantage aux ressources de la mémoire plutôt qu'à céder aux sirènes de modèles technocratiques devenus banals à force d'être copiés, fournissant des réponses souvent insuffisantes aux territoires auxquels ils s'appliquent. Ce choix est déjà fait par de nombreux territoires. Le passé devient porteur d'avenir. De nombreux territoires font désormais ce choix. Ainsi lorsque le Département de la Meuse proclame qu'il « est le département de la Grande Guerre »(Conseil Général de la Meuse, 2012), il exprime un volontarisme politique construit autour d'un pan de mémoire. Une option dont la limite est constituée par la multiplication de ce type de démarche : « La concurrence mémorielle est (...) un enjeu structurant et déterminant pour la cohésion sociale de nos sociétés " (Grandjean, Jamin, 2011).

\section{- La mémoire comme levier d'affirmation identitaire}

La mise en scène de la mémoire peut également viser un objectif avant tout social. Elle conforte la cohésion du groupe social inscrit dans un territoire. Cette mémoire peut ainsi consacrer la dimension éducative d'un lieu (monument, site, ville, nation...) disposant d'une valeur symbolique reconnue par un groupe social ou un ensemble de groupes sociaux. Cette dernière peut n'avoir qu'une valeur locale ou au contraire atteindre une portée nationale, européenne voire universelle... et disposer d'une puissance morale d'intensité variable (des notions de patrimoine à sauvegarder à celle de devoir de mémoire). La mémoire du territoire rassemble autour de valeurs et d'une identité commune. Intéressons nous à la notion d'identité. Celle-ci a connu un succès croissant, souvent associée à celle de culture. En même temps qu'ils mettaient en avant 
une culture propre, de nombreux acteurs ont ainsi témoigné, à partir des années 1960, du souci d'inscrire leur identité collective dans l'histoire, une histoire des vainqueurs et des dominants, au nom de leur mémoire de vaincus et de dominés, certes, mais aussi au nom de la contribution de leur groupe de référence à la culture et à la vie collective. Identités et mémoires, individuelles ou collectives sont devenus des thèmes inséparables. L'identité n'est possible que parce qu'il y a mémoire.

L'anthropologue Isaac Chiva définit l'identité comme « la capacité que possède chacun de nous de rester conscient de la continuité de sa vie à travers changements, crises, ruptures " (Augé M., 1992), tandis que pour le philosophe Paul Ricoeur (1992), parler d'identité, c'est parler du «maintien de soi à travers le temps ». Joël Candau (1998) précise que la mémoire précède la construction de l'identité, elle est un des éléments essentiels de sa recherche, qu'elle soit individuelle ou collective. Mémoire et identité sont indissociables et se renforcent. Selon Gilles Bataillon (2006), le « contexte » structure également les identités collectives et individuelles.

Enfin Michel Wieviorka définit l'identité collective comme l'ensemble des références culturelles sur lesquelles se fonde le sentiment d'appartenance à un groupe ou à une communauté, qu'elle soit réelle ou « imaginée » (Anderson B., 1996). Dans les sociétés contemporaines, ces références sont plus en plus nombreuses et conscientes, affirmées voir même revendiquées. Ces références sont perçues comme autant de productions sociales attribuées à un groupe humain, ou encore associées à une origine divine ou mythique. Une identité collective dessine non pas une série de significations ou un sens, mais un système de valeurs qui définit l'unité d'un groupe. L'identité s'organise donc en un ensemble de traits qui ne deviennent des orientations pour l'action qu'à partir du moment où elle se trouve confrontée à des défis, internes ou externes mais pas forcement conflictuels (Wieviorka M., 2001).

Les valeurs d'un groupe s'expriment selon une intensité variable, du factuel à la sanctuarisation du lieu. Par exemple le sang versé pour la patrie incarné par le Dôme des Invalides, la Zone rouge de Douaumont ou les cimetières militaires avec des croix blanches pour les alliés, des croix noires pour les Allemands, un carré musulman et un carré israélite regroupés en des lieux proches mais chacun à l'opposé du site de Douaumont. La reconstruction à l'identique de quartiers détruits pendant les guerres (la grand place de Tournai ou le centre de Dresde reconstruits après la seconde guerre mondial, le vieux Varsovie détruit lors du soulèvement de la ville en 1944, reconstruit à l'identique suite à un important effort de mobilisation nationale) répond aussi à ce besoin d'ancrage et de références historiques. Elle permet de se rassurer, de reconstruire le paysage familier et reconnu qui incarne dans un lieu l'identité des habitants qui s'y reconnaissent. Dans ce cas même le pastiche architectural s'oublie. Il est jugé préférable à l'oubli. La notion d'héritage culturel, patrimonial porteurs de valeurs identitaires affirmées à des moments clefs de l'histoire de l'espace en jeu et qu'il convient de consacrer. Quelle que soit son échelle, la " mise en mémoire » d'un territoire est plus ou moins accessoire (telle ville a son cimetière militaire) ou totale (villes de pèlerinage religieux, entièrement organisées, de façon active et prosélyte autour de cette fonction mémorielle et sacerdotale : Lourdes par exemple...). A un moindre échelon, lieux de mémoire militaires : Vimy, ND de Lorette, Douaumont, «Forêts de la mémoire » de l'Office National des Forêts (ONF, 2012). La mémoire nourrit le mythe fondateur qui confère sa territorialité à une portion d'espace géographique. 


\section{La mémoire comme levier de développement économique d'un territoire}

33 La valorisation de la mémoire s'apparente parfois à un moyen d'accroître la valeur d'un territoire sur les plans économique, foncier, immobilier, de l'image (en interne et à l'extérieur, sur le plan de l'attractivité touristique par exemple). L'objet mémoriel valorisé l'est avant tout en tant que moyen de rehausser la valeur ajoutée et l'attractivité économique d'un espace, quitte à ce que cette mémoire ne soit qu'un pastiche ou un mythe : le packaging et le marketing permettent alors de faire prévaloir la forme sur le fond. Les économistes parlent de "consommation patrimoniale" (Benhamou, 2012). Les ruines et monuments antiques de Rome, Carthage, Athènes ou du Machu Pichu attirent le touriste ; Le village du père-Noël en Finlande ou le Royaume enchanté de Disney donnent respectivement à Rovaniemi et à Marne-la-Vallée une centralité que ces villes n'avaient pas jusqu'alors tandis que le centre-ville historique de Pékin, partiellement reconstruit au point d'être plus «authentique » qu'il ne l'était avant sa destruction récente, répond aux attentes d'un public judicieusement ciblé par le marketing et qui vient y dépenser ses devises. La création de produits culinaires "typiques" vendus dans les aéroports de destinations exotiques (pâté d'ours à l'aéroport d'Helsinki par exemple, qu'une minorité de Finlandais seulement a consommé) contribuent à conforter l'originalité et par conséquent l'attractivité économique (en l'occurrence touristique) d'un espace en offrant aux visiteurs des produits qu'ils s'attendent à trouver consommés depuis toujours par la population locale.

Document $n^{\circ} 3$ : Postures de prise en compte de la mémoire dans l'aménagement

\begin{tabular}{|c|c|c|c|}
\hline & \multicolumn{2}{|c|}{ Comment? } \\
\hline & & Valoriser & Détruire \\
\hline \multirow{3}{*}{ 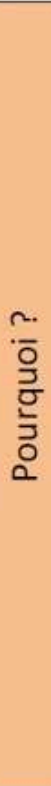 } & Se différencier & $\begin{array}{l}\text { Le Département de la Meuse affirme } \\
\text { qu'il « est le département de la Grande } \\
\text { Guerre " et met en place un } \\
\text { programme de valorisation territoriale } \\
\text { à base mémorielle avec l'appui sa } \\
\text { "Mission Histoire». }\end{array}$ & $\begin{array}{l}\text { Les vagues de destruction de formes } \\
\text { urbaines jugées banales ou obsolètes } \\
\text { à un moment donné : barres HLM, } \\
\text { anciennes usines, cités minières, } \\
\text { courées, corons. }\end{array}$ \\
\hline & Se rassembler & $\begin{array}{l}\text { La reconstruction à l'identique de } \\
\text { quartiers détruits pendant les guerres: } \\
\text { la Grand place de Tournai ou la ville du } \\
\text { Havre par exemple, reconstruites } \\
\text { après la seconde guerre mondiale. }\end{array}$ & $\begin{array}{l}\text { La destruction de monastères } \\
\text { tibétains par la Chine, des Bouddhas } \\
\text { de Damiyan par les Talibans, des } \\
\text { Eglises par les Révolutionnaires } \\
\text { Français de } 1789 . .\end{array}$ \\
\hline & Se développer & $\begin{array}{l}\text { Les ruines antiques de Rome, } \\
\text { Carthage, Athènes ou du Machu Pichu } \\
\text { mises en tourisme. Le village du père- } \\
\text { Noël à Rovaniemi en Finlande ou le } \\
\text { Royaume enchanté de Disney qui } \\
\text { confèrent à ces territoires } \\
\text { périphériques un rayonnement } \\
\text { économique. La recomposition } \\
\text { d'anciens quartiers industriels et leur } \\
\text { embourgeoisement : Docklands de } \\
\text { Londres, bientôt Newham après les Jo } \\
\text { de } 2012 \text { ? }\end{array}$ & $\begin{array}{l}\text { L'élimination des freins et obstacles au } \\
\text { développement économique: Doel } \\
\text { dans la périphérie portuaire d'Anvers, } \\
\text { absorbé par le développement } \\
\text { portuaire ou Holzweiler confronté à } \\
\text { l'exploitation du lignite en Rhénanie. }\end{array}$ \\
\hline
\end{tabular}

Document original réalisé par Ginet P.

C'est en suivant également cette logique que des maires investissent dans la Culture. Cependant la ou plutôt les mémoires, évoquent des territorialités multiples, à 
différentes échelles, en perpétuelle recomposition, porteuses de sens différents selon les individus qui les investissent au cours des périodes de la vie (Michaud, 2006) ceci dans le cadre d'un espace géographique appréhendé comme une métastructure sociospatiale, c'est-à-dire "l'espace immédiat autour de l'aire résidentielle, plus l'ensemble des coquilles du vécu " (Di Méo, 1991). La mise en valeur du patrimoine, souvent associée au secteur culturel, est devenue un élément incontournable des projets régionaux pour un renouveau économique et des programmes de renouveau urbain, notamment pour les territoires postindustriels tels que ceux d'Europe de l'Est ou du Nord-est Anglais. La disparition ou la restructuration des activités traditionnelles de ces territoires a entrainé de graves difficultés économiques et sociales. Ce tournant postindustriel et la reconstruction économique de ces territoires ont fortement influencé et modifié les perceptions du patrimoine. Dans un premier temps, la préservation ou la mise en valeur de l'héritage industriel étaient restées secondaires. Plusieurs possibilités : la destruction de l'ensemble du patrimoine dans le but de faire place pour implanter de nouvelles structures (ou dans le cadre d'un réaménagement urbain), ou pour gommer l'image industrielle ancienne et déliquescente du territoire (Bailoni, 2008). La promotion du patrimoine historique préindustriel et naturel est ensuite apparue dans les années 80-90, comme un bon outil pour donner une autre image à ces territoires que celles des mines et des usines en crise (Lanigan, 1999) jusqu'à en arriver parfois à l'effet «Bilbao ». Les notions de patrimoine et d'héritage culturel sont instrumentalisées dans les discours, alors que le territoire demeure en réalité profondément marqué par une culture ouvrière. Les acteurs locaux entendent fournir de nouveaux repères sociaux et identitaires aux habitants mais aussi à la société toute entière. Les constructions industrielles font l'objet d'un nouveau regard et ont alors été considérées, au même titre que des monuments plus anciens, comme des témoignages historiques qu'il convenait de préserver. Ce sont d'abord des historiens britanniques (Michael Rix, Robert Angus Buchanan, Kenneth Hudson) des universités de Birmingham, d'Edimbourg et de Hull qui ont pris conscience de l'intérêt de ce patrimoine industriel et minier (Deshaies, 2008). L'Allemagne (avec la "Route der Industriekultur" inaugurée en 1999), le Royaume-Uni et la Suède ont été précurseurs en ce qui concerne le Patrimoine minier et chacun de ces pays a au moins un ou deux sites miniers inscrits au Patrimoine mondial de l'Unesco. En France, ce n'est que beaucoup plus récent (2012: inscription du bassin minier du Nord-Pas-de-Calais).

Si les retombées d'un investissement mémoriel posent question, force est de constater qu'un véritable marché de la communication existe autour de cette thématique, comme en témoigne l'existence d'entreprises qui en ont fait leur fonds de commerce de prestations auprès de communes pour des projets urbains de valorisation territoriale par le biais d'installations signalétiques à portée historique (mise en place de panneaux qui exposent des photographies anciennes des lieux... de mémoire).

\section{Conclusion}

La notion de mémoire renvoie à la sphère de l'intime. Elle concerne chaque individu dans ce qu'il conserve en lui d'expériences personnelles passées, hiérarchisées en fonction de valeurs dictées par son «habitus»(Bourdieu, 2005). Elle reflète par conséquent tout autant l'individu qu'elle exprime les formes de sociabilité de plus en plus entremêlées dans lesquelles celui-ci se construit et peut évoluer tout au long de sa 
vie. La mémoire fonde par conséquent la culture et l'identité de chaque individu, de chaque groupe social. Elle en constitue l'armature sans laquelle ils vacillent et se perdent. Les valeurs qu'elle exprime et le rôle qu'elle joue dans les choix à faire, fussent-ils mythifiés, donnent à une partie de cette mémoire une dimension qui confine au sacré. La mémoire envisagée sous cet angle, dans les sociétés laïques, démocratiques, ouvertes et républicaines bouleversées par la mondialisation, est instable et fragile. Elle se nourrit de références multiples et changeantes et s'oriente parfois vers des choix radicaux, certains pans de mémoire se trouvant effacés et d'autres mis en exergue de manière exclusive. Yves Lacoste (1992) parle de représentations géopolitiques contradictoires lorsque des visions d'un territoire, de son histoire sont différentes voire même opposées.

L'instrumentalisation de la mémoire constitue par conséquent un enjeu géopolitique majeur. L'urbanisation accélérée de la planète et la mondialisation ont brassé et rassemblé sur quelques sites urbains, en quelques décennies seulement, des populations multiples qui ont amené avec elles leurs valeurs, leurs identités, leurs racines, leurs mémoires... Ce concept de mémoire, a priori plus polémique que fédérateur, plus sujet à controverse que moyen de rassembler, interroge par conséquent le politique dans son appréhension. L'enjeu concerne au moins le maintien de la paix sociale et de l'ordre républicain, au mieux, le partage d'un projet d'avenir commun. Pourtant les thèses s'affrontent. D'un côté celle de cultures et donc de mémoires immiscibles : S.Huntington et du choc des civilisations (Huntington, 1993), le nationalisme, le régionalisme militant (corse, basque...), autant de mémoires légitimant l'affrontement. De l'autre, celles de mémoires miscibles (sous-entendu sans heurts à l'instar de la civilisation gallo-romaine) au travers des concepts de métissage, de créolisation (Glissant, 1995), d'identité-rhizome (Deleuze, 1972) ou d'interculturalité. Les premières définissent des frontières, les secondes proposent leur dépassement.

L'aménageur et l'urbaniste, dans leur souci de conforter la territorialité de leur espace de réflexion et d'action et d'en garantir la cohésion, selon la doctrine en vigueur, sont amenés à intégrer de façon implicite ou explicite la question de la mémoire puisque " Le territoire est une appropriation à la fois économique, idéologique et politique (sociale, donc) de l'espace par des groupes qui se donnent une représentation particulière d'eux-mêmes, de leur histoire " (Di Méo, 1996). La mondialisation, l'individualisme, le brassage culturel et communautaire ont juxtaposé des groupes sociaux multiples qui ne se reconnaissent plus nécessairement dans les mêmes mémoires. Si la capacité à se servir de la mémoire comme levier de développement et de cohésion se trouve posée, elle est par contre un excellent révélateur des clivages voire des recoupements qui existent entre groupes sociaux, comme des valeurs que chacun d'eux souhaite conserver pour les générations futures. La question est de savoir s'il existe des éléments de mémoire partagés par les différents groupes sociaux et les différents acteurs de l'aménagement. Une sorte de fil rouge mémoriel qui emprunterait au passé et surtout, projetterait vers l'avenir les territoires en donnant sens à l'action d'aménagement, légitimée par des valeurs communes et partagées. 


\section{BIBLIOGRAPHY}

Anderson B., 2006, L'Imaginaire national. Réflexions sur l'origine et l'essor du nationalisme, Paris, La Découverte, 212p.

Association « Les Amis de la Fondation pour la Mémoire de la Déportation », www.afmd.asso.fr Audrerie D., 2003, Petit vocabulaire du patrimoine culturel et naturel. Ed. Confluences, Bordeaux, 63p. Augé M. (dir), 1992, Territoires de la mémoire, Thonon-les-Bains, Ed. de L’Albaron, 133p.

Bailoni M., 2008, «Quelle place pour le patrimoine dans le renouveau d'une région postindustrielle? Le cas du Nord-Est anglais ", Revue Géographique de l'Est, volume 48, Reconversion et patrimoine au Royaume-Uni : http://rge.revues.org/682

Beghain P., 2012, Patrimoine, politique et société, Paris, Presses de Sciences Po, 162p.

Benhamou F., 2012, Economie du patrimoine culturel, Paris, La découverte, 126p.

Bortolotto C. (dir), 2011, Le patrimoine culturel immatériel : enjeux d'une nouvelle catégorie, éd. De la Maison des Sciences de l'homme, 251p.

Bourdieu P., 2005, Le sens pratique, Paris, Editions de minuit, 474p.

Candau J., 1998, Mémoire et Identité, Paris, PUF, 225p.

Chesneaux J., 2001, Mémoire urbaine et projet urbain, Le quotidien urbain, 194p.

Choay F., 1965, L'urbanisme, utopies et réalités : Une anthologie, Paris, Seuil, 446p.

Choay F., 2009, Le patrimoine en question : anthologie pour un combat, Paris, éd. du Seuil, 214p.

Choay F., 1996, L’allégorie du patrimoine, Paris, éd. du Seuil, 260p.

Conseil Général de la Meuse, http://www.meuse.fr/page.php?url=culture-et-tourisme/memoirede-la-grande-guerre, le 07.07.12.

Crubellier M., 1991, La mémoire des français. Recherches d'histoire culturelle, Paris, Henry Veyrier et Kronos, 352p.

Deshaies M., 2008, «Les bassins houillers d'Europe : des paysages entre héritages et renouveau », Géoconfluence, http://geoconfluences

Deleuze G., Guattari F., 1972, Capitalisme et schizophrénie, Paris, Editions de Minuit, 354 p.

Di Méo G., 1998, Géographie sociale et territoire, Paris, Nathan, 317p.

Di Méo G., 1996, Les territoires du quotidien, Paris, L'Harmattan, 207p.

Di Méo G., 1991, L'homme, la société, l'espace, Paris, Anthropos, 319p.

Edelblutte S., 2009, « Paysages et territoires du patrimoine industriel au Royaume-Uni », Revue Géographique de l'Est, vol. 48 / 1-2 | 2008.

Galland O., Lemel Y., 2007, Valeurs et cultures en Europe, Paris, La découverte, 120 pages.

Glissant, E., 1995, Introduction à une poétique du divers, Paris, Gallimard, 147 p.

Gracq J., 1989, La forme d'une ville, Paris, Corti, 213p.

Grandjean G., Jamin J., 2011, La concurrence mémorielle, Paris, Colin, 256p. 
Gravari-Barbas M., Ripoll F., 2010, « Introduction : De l'appropriation à la valorisation, et retour », Revue Noroi, $\mathrm{n}^{\circ} 217$ «Interroger les processus de valorisation des espaces urbains », 118p.

Hayot A., Sauvage A. (dir.), Le projet urbain, Actes du colloque de Marseille. Ed. de la Villette, Paris, $2000,402 \mathrm{p}$

Heinich N., 2009, La Fabrique du patrimoine : « de la cathédrale à la petite cuillère », Paris, éd. de la Maison des Sciences de l'homme, 286p.

Hulbert F., 2009, «L'espace politique de la ville : plaidoyer pour une géopolitique urbaine », L'espace politique, http://espacepolitique.revues.org/index1330.html

Huntington S., 2007, Le choc des civilisations, Paris, O.Jacob, 402p.

Lacoste Y., 1995, Dictionnaire de Géopolitique, Paris, Flammarion, 1699p.

Lacoste Y., 1992, «Des représentations géopolitiques contradictoires », Matériaux pour l'histoire de notre temps, numéro 27. p37.

Lanigan C., 1999, Regionalism and Regional Identity in North East England, These, University of Newcastle-Upon-Tyne, 265 p.

La rédaction, «Editorial : Identité et action collective, Cultures \& Conflits », n¹2, hiver 1993 : http://conflits.revues.org/435

Lynch K., 1969, L'image de la cité, Paris, Dunod, 222p.

Michaud J-L., Girard-Hainz B., 2006, Mémoires de territoires, solidarités de voisinage et développement local. Paris, actes du colloque Territoires, action sociale et emploi, 22-23 juin, 14p.

Moles A., 1969, « Sociodynamique et politique d'équipement culturel dans la société urbaine », Communications, n14, p137.

Nora P., 1997, Les lieux de mémoire, Paris, Gallimard, 1652p.

Nora P., 2011, Présent, nation, mémoire, Paris, Gallimard, 418 pages.

Obin J-P., 2004, Les signes et manifestations d'appartenance religieuse dans les établissements scolaires, Paris, Ministère de l'éducation nationale, 38p.

Office National des Forêts, http://www.onf.fr/gestion_durable/sommaire/action_onf / patrimoine/20080625-103105-91656/index.html

Patin V., 2005, Tourisme et patrimoine, Paris, la documentation française, 207p.

Ricoeur P., 1992, Entre mémoire et histoire, Thonon-les-Bains, Ed. de l'Albaron, 139p.

Rosière S., 2007, Géographie politique \& Géopolitique, Paris, Ellipses, 426p.

Stora B., 1999, Le transfert d'une mémoire, Paris, La Découverte, 141p.

Subra P., 2007, Géopolitique de l'aménagement du territoire, Paris, Armand Colin, 327p.

Tadié Jean-Yves et Marc, Le sens de la mémoire, Paris, Gallimard, 1999, 356p.

Todorov T., 1995, Les abus de la mémoire, Paris, Arléa, 61p.

Trifon P., Goma P., Manea N., 2008 « Le témoignage littéraire dans l'engrenage de la concurrence mémorielle », Le courrier des Balkans, http://balkans.courriers.info/article10760.html

UMR PRODIG, http://geomonde.univ-paris1.fr/geomonde/4-notices.php, le 20.07.12.

UNESCO, 1972, Convention concernant la protection du patrimoine mondial culturel et naturel, Paris, $15 \mathrm{p}$. 
UNESCO, 2002, Le patrimoine culturel immatériel, miroir de la diversité culturelle, IIIème Table ronde des Ministres de la culture, Istanbul, $3 p$.

Veschambre V., 2008, Traces et mémoires urbaines, enjeux sociaux de la patrimonialisation et de la démolition, Rennes, PUR, $315 \mathrm{p}$.

Wieviorka M., 2001, La différence, Paris, Balland, 201p.

\section{ABSTRACTS}

The theme of memory is not much investigated by Geographers, unlike heritage, almost exclusively discussed in terms of its inventory and its conservation. The relationships among memory to the act of arranging space, whether implicit or clearly explained, are yet the expression of political choices that raise major geopolitical issues of interest to the geographer. This is especially true since their formulation is shaken today by globalization and urbanization brew populations of diverse origin in the maelstrom of the city. This phenomenon also affects gradually and increasingly small towns The manipulation of memory in this context raises the question of its value or its omission, the purpose of his economic and social identity, the ability to rally around a shared humanist project.

La thématique de la mémoire est peu investie par les Géographes, contrairement à celle du patrimoine, presque exclusivement abordée sous l'angle de son inventaire et de sa conservation. Les relations unissant la mémoire à l'acte d'aménager l'espace, qu'elles soient implicites ou clairement explicitées, constituent pourtant l'expression de choix politiques qui soulèvent des enjeux géopolitiques majeurs susceptibles d'intéresser le géographe. Ceci est d'autant plus vrai que leur formulation est bousculée de nos jours par une mondialisation et une urbanisation qui brassent des populations d'origine diverse dans le maelström de la grande ville. Ce phénomène touche également peu à peu et de manière croissante les petites villes. L'instrumentalisation de la mémoire soulève dans ce contexte la question de sa valorisation ou de son oubli, celle de sa finalité économique, identitaire et sociale, celle de sa capacité à rassembler autour d'un projet humaniste partagé.

Das Thema der Speicher nicht durch Geographen investiert, anders als Erbe, fast ausschließlich in Bezug auf ihren Bestand und ihre Erhaltung diskutiert. Die Beziehungen zwischen Speicher auf den Akt der Vermittlung Raum, ob implizite oder deutlich erklärt, sind noch Ausdruck des politischen Entscheidungen, die großen geopolitischen Fragen von Interesse für die Geographen erhöhen. Dies gilt insbesondere, da ihre Formulierung ist erschüttert heute durch die Globalisierung und Urbanisierung Gebräu Populationen unterschiedlicher Herkunft in den Sog der Stadt. Die Manipulation der Speicher in diesem Zusammenhang stellt sich die Frage ihres Wertes oder ihrer Unterlassung, der Zweck seiner wirtschaftlichen und sozialen Identität, die Fähigkeit, um eine gemeinsame humanistische Projekt zu sammeln.

\section{INDEX}

Mots-clés: aménagement, géopolitique, identité, mémoire, valeurs

Keywords: geopolitics, identity, memory, planning, values

Schlüsselwörter: Entwicklung, Geopolitik, Identität, Speicher, Werte 
AUTHORS

PIERRE GINET

Professeur, Chercheur associé au Laboratoire Discontinuités (EA2468)

LAURĖNE WIESZTORT

Docteure en géographie, laboratoire Discontinuités (EA2468) 